\title{
Revisiting the mass-luminosity relation with an effective temperature modifier
}

\author{
Jifei Wang ${ }^{1}$ and Zehao Zhong ${ }^{1,2}$ \\ 1 Key Laboratory of Optical Astronomy, National Astronomical Observatories, Chinese Academy of Sciences, \\ Beijing 100101, PR China \\ e-mail: wjf@zihexin.com \\ 2 School of Astronomy and Space Science, University of Chinese Academy of Sciences, Beijing 100049, PR China \\ e-mail: zhzhong@nao.cas.cn
}

Received 19 August 2018 / Accepted 27 September 2018

\begin{abstract}
The mass-luminosity relation (MLR) is commonly used to estimate the stellar mass. The classical MLR can hardly fit data of all the stellar mass range, thus researchers have generally adopted piecewise MLRs based on the classical MLR with different exponents for different mass ranges. However, varying turning points for the piecewise MLRs and for the exponent of each segment were used, and the estimated stellar masses are not always as good as those obtained by dynamical methods. We suggest an alternative way to improve the mass estimation accuracy: adding an effective temperature modifier to modify every segment MLR. We use a corresponding estimating equation for G- and K-type main-sequence stars, and verify this equation on two eclipsing binary catalogs. We compare the estimated results with those from a classical MLR and several piecewise MLRs. We find that the new estimates are significantly more accurate than those from the classical MLR and some piecewise MLRs, and they are not inferior to the stellar masses from other piecewise MLRs. This indicates that the temperature modifier can effectively help improve the estimation accuracy. In addition, we discuss the effect of adding the temperature modifier on the practicability of estimating stellar masses.
\end{abstract}

Key words. binaries: eclipsing - stars: fundamental parameters

\section{Introduction}

As a fundamental physical quantity, stellar mass plays an important role in stellar physics and the dynamics of stellar systems, for instance. However, the vast majority of stellar masses are difficult to measure directly and are often estimated using the mass-luminosity relation (MLR). Determining the MLR requires accurate and reliable data on stellar mass, mainly from binary star systems, especially detached eclipsing binaries (Andersen 1991; Torres et al. 2010). Asteroseismology can also help in obtaining precise stellar masses (North et al. 2007, 2009), but so far, there are only few data sets. In addition, the total luminosity is needed, but we were unable to directly observe the total luminosity of a star. Some researchers have therefore replaced the total luminosity with a single-band absolute magnitude, and drew the massmagnitude relation. For example, Henry \& McCarthy (1993) obtained the mass-magnitude relation of 0.08-2 solar-mass stars for the $V, J, H, K$ bands. Benedict et al. (2016) used astrometry combined with radial velocity data to obtain the mass-magnitude relation of $0.08-0.62$ solar-mass stars for the $V, K$ bands. In addition, if the effective temperature $T_{\text {eff }}$ and radius $R$ of a star are known, the total luminosity $L$ of the star can be calculated directly, using the equation $L=4 \pi R^{2} \sigma T_{\text {eff }}^{4}$ ( $\sigma$ is the Stefan-Boltzmann constant). Otherwise, the star's apparent magnitude can be converted into bolometric magnitude to calculate the stellar luminosity by means of bolometric correction (Flower 1996).

In the early stage, classical MLR, such as $L \propto M^{\alpha}$ is used to fit the observed data $M$ means stellar mass and $\alpha$ represents the fitting exponent). One single logarithmic linear MLR can hardly fit the stellar mass with luminosity of the entire mass range, however, because the internal structure and the main way of producing energy may not be the same for stars of different masses. In this case, piecewise MLRs are adopted to solve this problem. For each segment of a piecewise MLR, the form of the classical MLR is commonly used, but with diverse exponents. However, various turning points have been used for the piecewise MLRs and also for exponents of different mass ranges. Table 1 shows three piecewise MLRs from different works. Griffiths et al. (1988) reselected the binary samples from Popper (1980), and drew their piecewise MLR in three sections. Demircan \& Kahraman (1991) discussed MLRs from models and from empirical data; Table 1 only shows the empirical MLR. Eker et al. (2015) used 268 high-precision data of detached eclipsing binaries to study the MLR. They split the MLR into four sections. This shows that not only the mass ranges and exponents, but also the number of segments may be different among researchers. For low-mass main-sequence stars, the exponent $\alpha$ is currently commonly around 4 .

The most important application of the MLR is to quickly compute the mass of a single star. However, the accuracy of estimated masses from one single classical MLR or piecewise logarithmic linear MLRs is often poor. We therefore revisit the MLR and attempt to improve the accuracy of the mass-estimating equation from the MLR. In our view, a piecewise equation seems to be necessary, but the logarithmic linear equations are not. Modifying every segment MLR by adding $T_{\text {eff }}$ modifiers based 
Table 1. List of piecewise MLRs from different works (of the form $\left.L \propto M^{\alpha}\right)$

\begin{tabular}{lcrc}
\hline \hline Name & Mass range (in $M_{\odot}$ ) & Exponent $\alpha$ & References \\
\hline \multirow{2}{*}{ G88 } & $M<0.40$ & 2.440 & $(1)$ \\
& $0.40<M<5.01$ & 4.160 & \\
& $M>5.01$ & 3.510 & \\
\hline D91 $^{a}$ & $0.10<M<18.10$ & 3.704 & $(2)$ \\
& $M<0.70$ & 2.616 & \\
& $M>0.70$ & 3.916 & \\
\hline E15 & $0.38<M \leqslant 1.05$ & 4.841 & $(3)$ \\
& $1.05<M \leqslant 2.40$ & 4.328 & \\
& $2.40<M \leqslant 7.00$ & 3.962 & \\
& $7.00<M<32.00$ & 2.762 & \\
\hline
\end{tabular}

Notes. ${ }^{(a)}$ MLR from empirical data.

References. (1) Griffiths et al. (1988); (2) Demircan \& Kahraman (1991); (3) Eker et al. (2015).

on the classical MLR might yield good results as well. We used data of two eclipsing binary catalogs to test this approach. In Sect. 2 we introduce the data selection. Section 3 displays the modifier and presents a comparison of different stellar mass estimations. We conclude in Sect. 4.

\section{Data selection}

To fit an MLR, stars with relatively reliable masses, radii, and effective temperatures are required. The most commonly used such stars are the eclipsing binary systems, and we took the detached double-line eclipsing binaries of the Milky Way (Eker et al. 2014) and DEBcat (Southworth 2015) as a primary sample.

The detached double-line eclipsing binaries of the Milky Way, also namely catalog of stellar parameters from detached double-line eclipsing binaries in the Milky Way (EKE14; Eker et al. 2014), is a catalog of 472 stars (236 binaries) with mass and luminosity parameters. The mass range is $0.18 M_{\odot} \sim$ $32.60 M_{\odot}$, the radius range is $0.21 R_{\odot} \sim 21.19 R_{\odot}$, and the temperature range is $2750 \mathrm{~K} \sim 43000 \mathrm{~K}$.

The full name of the DEBcat is catalog of the physical properties of well-studied eclipsing binaries. It is constantly updated over the years. As of February 28, 2018, the catalog contains 398 stars (199 binaries). The ranges of mass, radius, and the temperature are $0.11 M_{\odot} \sim 27.27 M_{\odot}, 0.21 R_{\odot} \sim 67.62 R_{\odot}$, and $2851 \mathrm{~K} \sim 38019 \mathrm{~K}$, respectively.

First we selected the main-sequence stars from the primary sample. Habets \& Heintze (1981) used the precise stellar parameters of their double-line spectroscopic binaries for a luminosity classification, and they showed the $\log g-\log M$ relationship at different luminosity classes, as displayed in Table 2 ( $g$ represents the surface gravity). The luminosity classifications $E V_{\odot}, V_{\odot}-V, V-I V$ express the boundaries of the extreme zero-age main sequence (ZAMS), between the ZAMS and the main sequence, and between the main sequence and giants, respectively, while $V_{\odot}$ and $V$ express the linear least-squares fitting of ZAMS stars and main-sequence stars in the sample of Habets \& Heintze (1981). We placed the stellar $\log M$ in our sample into the corresponding function, and calculated the theoretical $\log g$, then we compared it with the real value of the sample. When $\log g_{V-I V} \leqslant \log g \leqslant \log g_{E V_{\odot}}$, we can select the main-sequence stars in the primary sample. Figure 1 displays
Table 2. Luminosity classification and corresponding fitting functions between $\log g$ and $\log M$ (Habets \& Heintze 1981).

\begin{tabular}{cc}
\hline \hline LC & Functions \\
\hline$E V_{\odot}$ & $\log g_{E V_{\odot}}=(-0.482 \pm 0.017) \log M / M_{\odot}+(4.711 \pm 0.016)$ \\
$V_{\odot}$ & $\log g_{V_{\odot}}=(-0.415 \pm 0.044) \log M / M_{\odot}+(4.610 \pm 0.031)$ \\
$V_{\odot}-V$ & $\log g_{V_{\odot}-V}=(-0.356) \log M / M_{\odot}+4.512$ \\
$V$ & $\log g_{V}=(-0.291 \pm 0.027) \log M / M_{\odot}+(4.337 \pm 0.016)$ \\
$V-I V$ & $\log g_{V-I V}=(-0.218) \log M / M_{\odot}+4.170$ \\
\hline
\end{tabular}

the picture of $\log g$ vs $\log M$ of the main-sequence star sample. The lines are functions of $E V_{\odot}, V_{\odot}, V_{\odot}-V, V-I V$, and $V$ in Table 2. Black solid points in (a) are stars from EKE14 ( MWEB stands for Milky Way eclipsing binaries), and blue circles in (b) are stars from DEBcat.

The logarithmic MLR of the sample stars is displayed in Fig. 2. In this figure, the MLR of this sample has several turning points, but they are not obvious, and it is difficult to obtain a uniform linear MLR. Although the exponent $\alpha$ remains around 4 , it does change in different mass ranges. Because of the relatively many G- and K-type stars in the sample, we only discuss the modification equations of $\mathrm{G}$ - and $\mathrm{K}$-type main-sequence stars. According to Table 7 in Habets \& Heintze (1981), the effective temperature range of G- and K-type stars is $3700 \mathrm{~K}<$ $T_{\text {eff }}<6050 \mathrm{~K}$. We used this condition to select the G- and K-type main-sequence stars in Fig. 2. There are 92 stars from EKE14 and 70 stars from DEBcat. There is some overlap of G- and Ktype main-sequence stars between EKE14 and DEBCat, but the differences in their parameters are very small. Since we cannot be sure which catalog is more accurate, we did not remove the overlap stars. Our final sample therefore contains 162 stars from EKE14 and DEBCat.

\section{Modified estimation and accuracy comparison}

We aim to improve the accuracy of the mass-estimating equation from the MLR. An alternative way might be to add a modifier based on the classical mass-estimating equation. According to the stellar luminosity equation $L=4 \pi R^{2} \sigma T_{\text {eff }}^{4}, L$ is proportional to the fourth power of $T_{\text {eff }}$ and the square of $R$, which means that it is more sensitive to changes in $T_{\text {eff }}$. In addition, in actual observations, the measurement of $R$ depends on an accurate distance measurement, but the measurement of $T_{\text {eff }}$ does not, which is more practical. Therefore, adding a $T_{\text {eff }}$ modifier may lead to a better mass-estimating equation without losing practicability.

For the classical MLR we set $\alpha=4$, combined with the stellar luminosity equation. We can easily derive the following Eq. (1), where $M_{0}$ represents the classically estimated stellar mass,

$\frac{M_{0}}{M_{\odot}}=\left(\frac{L}{L_{\odot}}\right)^{\frac{1}{4}}=\frac{T_{\text {eff }}}{T_{\odot}}\left(\frac{R}{R_{\odot}}\right)^{\frac{1}{2}}$.

We envision building a simple enough modifier to modify Eq. (1). After some trials, we found one kind of appropriate modifier, $\beta$, and the modification equation is as bellow, where $M_{\beta}$ represents the modified estimated mass:

$\frac{M_{\beta}}{M_{\odot}}=\frac{M_{0}}{M_{\odot}} \cdot \beta=\left(\frac{L}{L_{\odot}}\right)^{\frac{1}{4}}\left(\frac{1}{3}\right)^{\left(\frac{T_{\mathrm{eff}}}{T_{\odot}}\right)^{\frac{1}{3}}-1}$.

We used Eq. (2) to estimate the modification stellar masses of $\mathrm{G}$ - and K-type main-sequence stars, and the results are shown in 


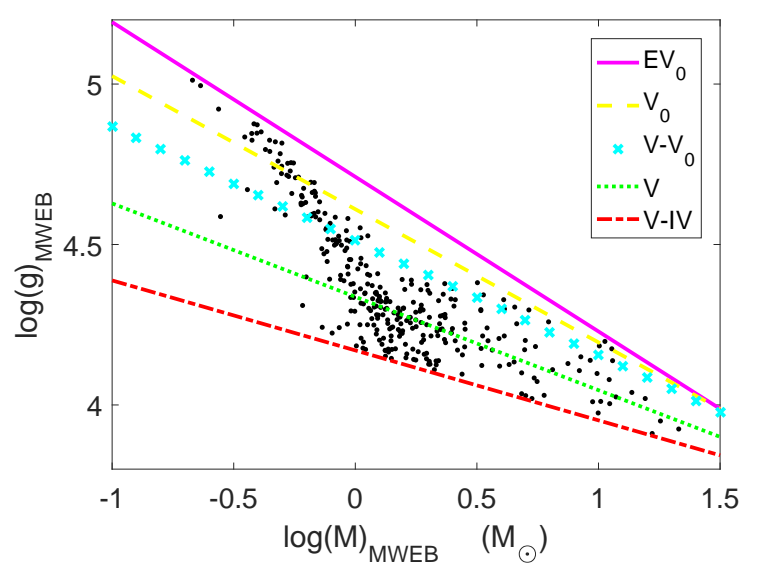

(a)

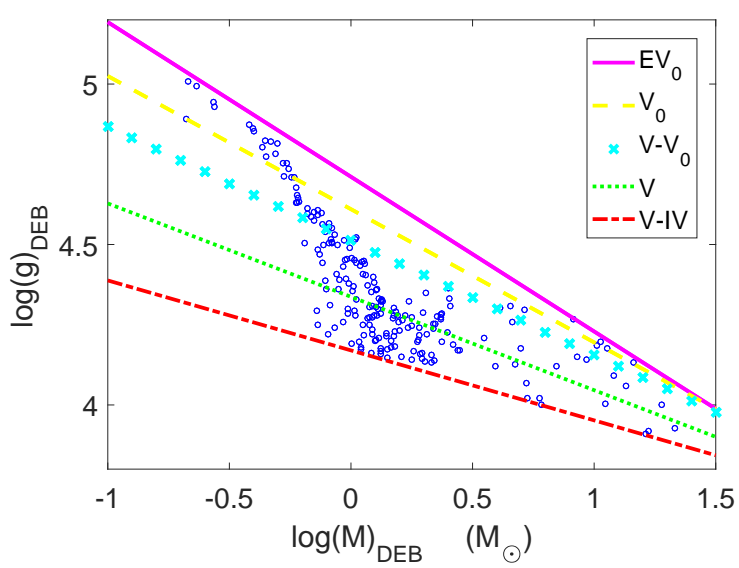

(b)

Fig. 1. Main-sequence stars selected from (panel a) EKE14 (black solid points) and (panel $b$ ) DEBcat (blue circles) according to the luminosity classification of Habets \& Heintze (1981). The straight lines from top to bottom are functions of $E V_{\odot}, V_{\odot}, V_{\odot}-V, V-I V$, and $V$ in Table 2 .

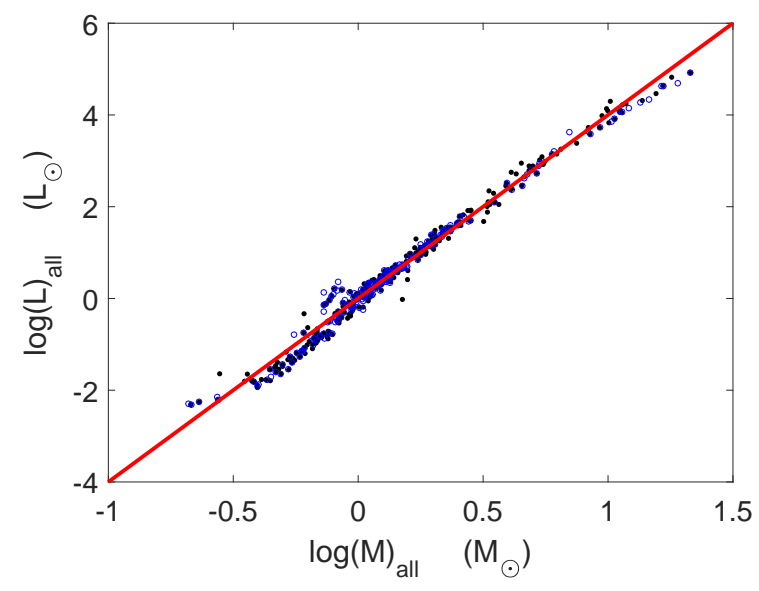

Fig. 2. Logarithmic MLR of main-sequence stars. Black solid points show stars from EKE14 and blue circles show stars from DEBcat. The red straight line represents one kind of classical MLR, $M \propto L^{\frac{1}{4}}$ for comparison.

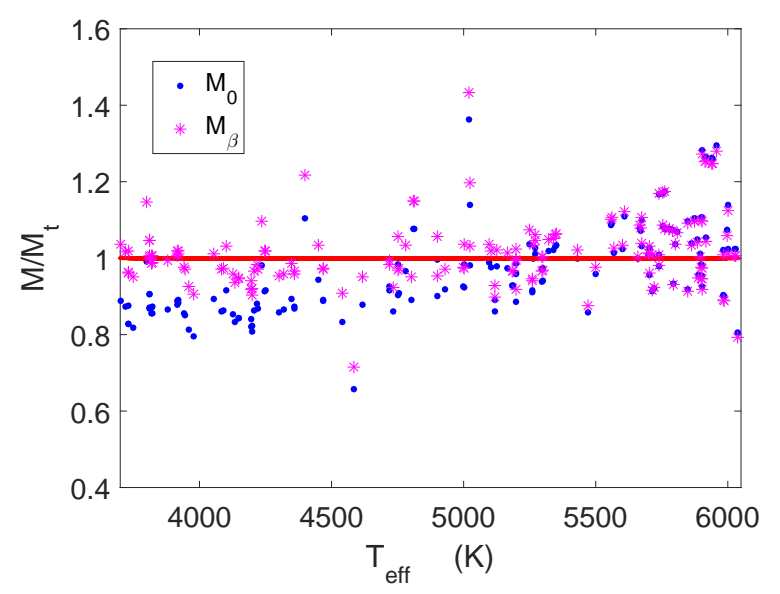

Fig. 3. Ratios of $M_{0} / M_{t}$ and $M_{\beta} / M_{t}$ varying with $T_{\text {eff }}$. Blue solid points show classically estimated masses, and magenta asterisks represent $\beta$ modification estimations.

Fig. 3, which displays the accuracy of classical and $\beta$ modification estimations that vary with $T_{\text {eff }} . M_{t}$ represents the dynamical stellar mass of the sample, substituting the true stellar mass for

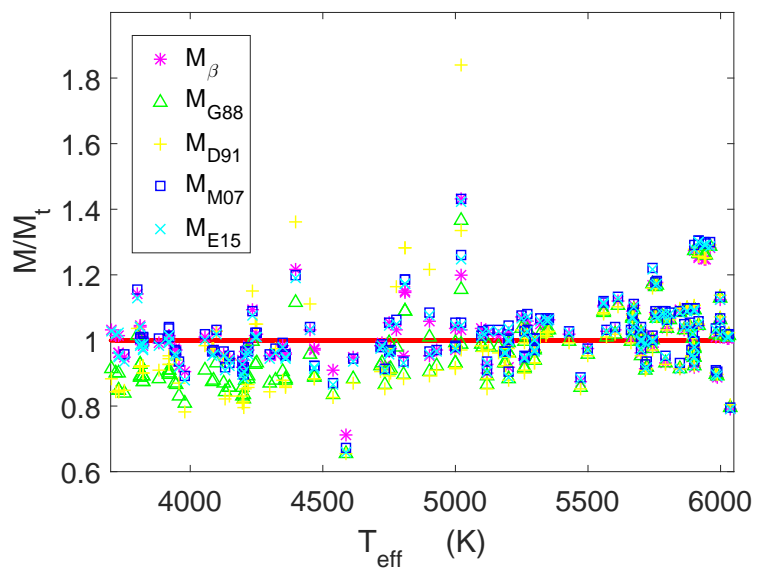

Fig. 4. Ratios of different estimated stellar masses to sample dynamical masses varying with $T_{\text {eff }}\left(M_{\beta}\right.$ is shown as magenta asterisks, $M_{\mathrm{G} 88}$ as green triangles, $M_{\mathrm{D} 91}$ as yellow pluses, $M_{\mathrm{M} 07}$ as blue squares, and $M_{\mathrm{E} 15}$ as indigo crosses).

comparison. Then $M / M_{t}$ can indicate the accuracy of the estimation. In this figure, compared with $M_{0}$, most $M_{\beta}$ are obviously more accurate for $T_{\text {eff }}<5300 \mathrm{~K}$, except for a few points. These stars are also poorly fit from other mainstream MLRs in Fig. 4. This is probably not the fault of modifier, but these stars do not seem to satisfy any MLRs, probably because they have relative large observational errors. On the whole, the modifier $\beta$ can indeed increase the accuracy of the classical estimating equation for $\alpha=4$. However, changing exponents of a piecewise MLR can also lead to a more accurate result. Therefore, the next step is to compare the accuracy of the estimated masses obtained by piecewise MLRs with our temperature modification MLR.

Malkov (2007) has studied the MLR of intermediate-mass stars, from which the mass estimating equation is $\log m=$ $0.00834+0.213 \log L+0.0107 \log ^{2} L$, for $-1.2<\log L / L_{\odot}<5.3$. We used the MLR of Malkov (2007) and three piecewise MLRs from Table 1 (Griffiths et al. 1988; Demircan \& Kahraman 1991; Eker et al. 2015) to estimate stellar masses of our final sample, and then labeld these estimated masses $M_{\mathrm{G} 88}, M_{\mathrm{D} 91}, M_{\mathrm{M} 07}$, and $M_{\mathrm{E} 15}$, respectively. The comparison result is displayed in Fig. 4. As can see in this figure, the accuracies of $M_{\mathrm{G} 88}$ and $M_{\mathrm{D} 91}$ are relatively low, and the accuracies of $M_{\mathrm{M} 07}$ and $M_{\mathrm{E} 15}$ are roughly similar to $M_{\beta}$. 
Table 3. Average values, standard deviations of the ratios of $M_{0}, M_{\beta}, M_{\mathrm{G} 88}, M_{\mathrm{D} 91}, M_{\mathrm{M} 07}$, and $M_{\mathrm{E} 15}$ to sample masses, and the percentages of stars within the corresponding relative error ranges.

\begin{tabular}{ccccccc}
\hline \hline Name & Average value & Standard deviation & $\mathrm{RE}<1 \%$ & $\mathrm{RE}<3 \%$ & $\mathrm{RE}<5 \%$ & $\mathrm{RE}<10 \%$ \\
\hline$M_{0} / M_{t}$ & 0.9679 & 0.1136 & $5.56 \%$ & $21.60 \%$ & $30.86 \%$ & $54.94 \%$ \\
$M_{\beta} / M_{t}$ & 1.0170 & 0.0915 & $11.73 \%$ & $33.95 \%$ & $55.56 \%$ & $83.95 \%$ \\
$M_{\mathrm{G} 88} / M_{t}$ & 0.9720 & 0.1084 & $8.64 \%$ & $22.22 \%$ & $30.86 \%$ & $62.35 \%$ \\
$M_{\mathrm{D} 91} / M_{t}$ & 0.9874 & 0.1372 & $6.79 \%$ & $21.60 \%$ & $32.72 \%$ & $61.73 \%$ \\
$M_{\mathrm{M} 07} / M_{t}$ & 1.0251 & 0.1013 & $9.88 \%$ & $27.78 \%$ & $48.15 \%$ & $79.63 \%$ \\
$M_{\mathrm{E} 15} / M_{t}$ & 1.0149 & 0.0997 & $8.64 \%$ & $30.25 \%$ & $52.47 \%$ & $81.48 \%$ \\
\hline
\end{tabular}

For quantitative comparison, Table 3 shows the average values, standard deviations of ratios of $M_{0}, M_{\beta}, M_{\mathrm{G} 88}, M_{\mathrm{D} 91}, M_{\mathrm{M} 07}$, and $M_{\mathrm{E} 15}$ to sample masses, and the percentages of stars within the corresponding relative error (RE) range. According to this table, although average value of $M_{\beta} / M_{t}$ is not the best, its standard deviation is the lowest and has the highest percentage of stars within any of the corresponding RE ranges. In addition, the percentages of stars for $M_{\mathrm{G} 88}$ and $M_{\mathrm{D} 91}$ are both relatively low, while the percentages of stars for $M_{\mathrm{M} 07}$ and $M_{\mathrm{E} 15}$ are slightly lower than for $M_{\beta}$ in every RE range. To summarize, we are conservative in thinking that the accuracy of our $\beta$ modification estimating equation is obviously higher than those of Griffiths et al. (1988) and Demircan \& Kahraman (1991), and is not inferior to those of Malkov (2007) or Eker et al. (2015). All the above indicates that the modifier $\beta$ can effectively help improve the accuracy of the stellar mass-estimating equation, which is an alternative way to changing exponents of a piecewise MLR. We also considered the practicability, and adding the temperature modifier does not affect the practicability of estimating the masses of stars (see Appendix A).

\section{Conclusions}

The MLR is one of the most basic properties of stars, and it can be used to estimate the mass of a single star when it is combined with a photometry measurement. One single classical MLR fitting cannot accomodate the entire mass range, which is the reason why logarithmic linear piecewise equations with different exponents have been adopted to improve the mass estimation accuracy. We suggest that adding the $T_{\text {eff }}$ modifier on the basis of the classical MLR can be an alternative way to improve estimation accuracy. We presented a new mass-estimating equation with the temperature modifier $\beta$ for G- and K-type mainsequence stars, and verified this approach on samples in two eclipsing catalogs, the EKE14 and DEBCat. We compared the $\beta$ modification estimations with the classical mass estimations and those from some mainstream MLRs. The results show that $\beta$ modification estimations have the lowest standard deviation and the highest percentages of stars within any of the corresponding $\mathrm{RE}$ ranges. This shows that the accuracy of the $\beta$ modification estimating equation is obviously higher than those of some mainstream MLRs, and is not inferior to the other MLRs. This means that using the temperature modifier to revise the massestimating equation can effectively improve the accuracy of stellar mass estimations. We also discussed methods for measuring the effective temperature of a star (see Appendix A). Choosing the right approach can help to quickly compute stellar effective temperatures in different situations. Therefore, we do not consider that the addition of temperature modifier would affect the practicability of estimating stellar mass.

We still think piecewise MLR should be adopted, but their turning points are still not agreed among researchers. More to the point, how many turning points are needed? It also remains debated whether they are necessary or if the trend of the MLR changes continuously and slowly, without specific turning points. In addition, the modifier we obtained can improve the accuracy of estimating the G- and K-type main-sequence stars, but for other spectral type stars, the modifier should be different. Furthermore, even with the G- and K-type main-sequence stars, the modifier $\beta$ may not be the best form. We suspect, however, that the $T_{\text {eff }}$ modifier may be related to the physical processes of the early stage of main-sequence stars, and may help to establish a more sophisticated physical model of ZAMS stars.

Acknowledgements. We thank Zhao Gang, Liu Jifeng, Jiang Xiaojun, and Wu Zhenyu for useful discussions.

\section{References}

Alonso, A., Arribas, S., \& Martinez-Roger, C. 1996, A\&A, 313, 873 Andersen, J. 1991, A\&ARv, 3, 91

Benedict, G. F., Henry, T. J., Franz, O. G., et al. 2016, AJ, 152, 141

Blackwell, D. E., \& Shallis, M. J. 1977, MNRAS, 180, 177

Blackwell, D. E., Petford, A. D., Arribas, S., Haddock, D. J., \& Selby, M. J. 1990, A\&A, 232, 396

Boyajian, T. S., McAlister, H. A., van Belle, G., et al. 2012a, ApJ, 746, 101

Boyajian, T. S., von Braun, K., van Belle, G., et al. 2012b, ApJ, 757, 112

Casagrande, L., Portinari, L., \& Flynn, C. 2006, MNRAS, 373, 13

Casagrande, L., Ramírez, I., Meléndez, J., Bessell, M., \& Asplund, M. 2010, A\&A, 512, A54

Demircan, O., \& Kahraman, G. 1991, Ap\&SS, 181, 313

Eker, Z., Bilir, S., Soydugan, F., et al. 2014, PASA, 31, e024

Eker, Z., Soydugan, F., Soydugan, E., et al. 2015, AJ, 149, 131

Flower, P. J. 1996, ApJ, 469, 355

González Hernández, J.I., \& Bonifacio, P. 2009, A\&A, 497, 497

Griffiths, S. C., Hicks, R. B., \& Milone, E. F. 1988, JRASC, 82, 1

Habets, G. M. H. J., \& Heintze, J. R. W. 1981, A\&AS, 46, 193

Henry, T. J., \& McCarthy, Jr., D. W. 1993, AJ, 106, 773

Kervella, P., Thévenin, F., Di Folco, E., \& Ségransan, D. 2004, A\&A, 426, 297

Kervella, P., Mérand, A., Pichon, B., et al. 2008, A\&A, 488, 667

Malkov, O. Y. 2007, MNRAS, 382, 1073

North, J. R., Davis, J., Bedding, T. R., et al. 2007, MNRAS, 380, L80

North, J. R., Davis, J., Robertson, J. G., et al. 2009, MNRAS, 393, 245

Popper, D. M. 1980, ARA\&A, 18, 115

Ramírez, I., \& Meléndez, J. 2005a, ApJ, 626, 446

Ramírez, I., \& Meléndez, J. 2005b, ApJ, 626, 465

Southworth, J. 2015, in Living Together: Planets, Host Stars and Binaries, eds.

S. M. Rucinski, G. Torres, \& M. Zejda, ASP Conf. Ser., 496, 164

Torres, G., Andersen, J., \& Giménez, A. 2010, A\&ARv, 18, 67 


\section{Appendix A: Effect of the $T_{\text {eff }}$ modifier on practicability}

When the modification equation is used to estimate the mass of stars, it is most important to measure the effective temperature of the star. There are many methods for measuring temperature, such as direct, indirect, and semi-direct methods. First, the direct method is not directly measuring the temperature, but directly measuring the stellar radius. Interferometry is one of the most important ways to do this (Kervella et al. 2004, 2008). Combined with the stellar luminosity, we can use the stellar radius as obtained from interferometry to compute the stellar effective temperatures. Unfortunately, the number of stars in interferometry is too small, and for large data samples, it is impossible to measure the radii of all stars with an interferometric approach. It is therefore necessary to combine interferometric measurements with the color or spectral types of stars and to establish empirical formulas for calculating effective temperatures. Boyajian et al. (2012) measured the radii of 44 A-, F-, and G-type stars by interferometry, and obtained their effective temperatures by combining the results with bolometric flux measurements. Based on these stellar parameters, they obtained the empirical equations of spectral-type- $T_{\text {eff }}$ and color $-T_{\text {eff }}$ for these stars. In the same way, Boyajian et al. (2012) measured the radii and effective temperatures of $21 \mathrm{~K}$ - and M-type stars by interferometry and bolometric flux measurements. They also obtained the empirical relation of $T_{\text {eff }}-$ color- $[\mathrm{Fe} / \mathrm{H}]$ for these stars. These empirical equations can be used to calculate the effective temperature of the stars in other samples.

Second, effective temperatures can be indirectly measured using the stellar spectral lines. The more common methods are the excitation equilibrium of the Fe lines, or fitting Balmer lines. The advantage of these two methods is that they are not affected by reddening, and the disadvantage is that they strongly depend on the model assumptions. In actual measurements, if there is a spectrum with a high signal-to-noise ratio $(\mathrm{S} / \mathrm{N})$, the temperature of the star can be measured by the above two methods, and the modification equations can be used to obtain the stellar mass.
In addition to directly measuring radius or indirectly using spectral lines, a so-called semi-direct method has often been used, called infrared flux method (IRFM), to calculate the effective temperature. The IRFM was first used by Blackwell \& Shallis (1977), and after 13 years of development, the theory was finally completed by Blackwell et al. (1990). The advantage of the this method is that it can simultaenously measure the angular diameter and the effective temperature of the star. The IRFM has frequently been used to measure the temperature of stars of different spectral types, and the $T_{\text {eff }}-$ color $-[\mathrm{Fe} / \mathrm{H}]$ relations of the stars were obtained accordingly (Alonso et al. 1996; Ramírez \& Meléndez 2005a,b; Casagrande et al. 2006, 2010; González Hernández \& Bonifacio 2009). The relations in these works are in the form of Eq. (A.1),

$\theta_{\mathrm{eff}}=a_{0}+a_{1} X+a_{2} X^{2}+a_{3} X[\mathrm{Fe} / \mathrm{H}]+a_{4}[\mathrm{Fe} / \mathrm{H}]+a_{5}[\mathrm{Fe} / \mathrm{H}]^{2}$.

Here, $\theta_{\mathrm{eff}}=5040 / T_{\mathrm{eff}}$, and $X$ represents the color of a star, and $a_{i}(i=0, \ldots, 5)$ are the fitting factors. Equation (A.1) can be used to calculate the stellar effective temperature as long as the photometry color and the $[\mathrm{Fe} / \mathrm{H}]$ data are known. Because the fitting factors $a_{i}$ are generally small, the error of $[\mathrm{Fe} / \mathrm{H}]$ has little effect on the error of the effective temperature. Even if the spectral $\mathrm{S} / \mathrm{N}$ is not high, if the accuracy of the photometry is high, the calculated effective temperature error will be relatively small.

In general, there are many ways to measure the effective temperature. For a spectrum with high $\mathrm{S} / \mathrm{N}$, the stellar temperature can be measured by the excitation equilibrium of the Fe lines, or by fitting Balmer lines. If only photometry and $[\mathrm{Fe} / \mathrm{H}]$ can be used, we can take advantage of the $T_{\text {eff }}-$ color- $-[\mathrm{Fe} / \mathrm{H}]$ relations to calculate the effective temperature. Even if photometry alone is available, using the empirical color- $T_{\text {eff }}$ relation can also help to calculate effective temperature. The greatest advantage in estimating stellar mass using the MLR is that a large sample of stars can be computed fast, and the methods we described above are all applicable to the rapid computing of large samples of stars, so that the addition of a temperature modifier does not affect the practicability of estimating the stellar masses. 